\title{
Improvement of phylogenetic method to analyze compositional heterogeneity
}

\author{
Zehua Zhang ${ }^{1}$, Kecheng Guo ${ }^{1}$, Gaofeng Pan ${ }^{1}$, Jijun Tang ${ }^{1,2}$ and Fei Guo ${ }^{\text {** }}$ \\ From The 10th International Conference on Systems Biology (ISB 2016) \\ Weihai, China.19-22 August 2016
}

\begin{abstract}
Background: Phylogenetic analysis is a key way to understand current research in the biological processes and detect theory in evolution of natural selection. The evolutionary relationship between species is generally reflected in the form of phylogenetic trees. Many methods for constructing phylogenetic trees, are based on the optimization criteria. We extract the biological data via modeling features, and then compare these characteristics to study the biological evolution between species.

Results: Here, we use maximum likelihood and Bayesian inference method to establish phylogenetic trees; multi-chain Markov chain Monte Carlo sampling method can be used to select optimal phylogenetic tree, resolving local optimum problem. The correlation model of phylogenetic analysis assumes that phylogenetic trees are built on homogeneous data, however there exists a large deviation in the presence of heterogeneous data. We use conscious detection to solve compositional heterogeneity. Our method is evaluated on two sets of experimental data, a group of bacterial 165 ribosomal RNA gene data, and a group of genetic data with five homologous species.

Conclusions: Our method can obtain accurate phylogenetic trees on the homologous data, and also detect the compositional heterogeneity of experimental data. We provide an efficient method to enhance the accuracy of generated phylogenetic tree.
\end{abstract}

Keywords: Phylogenetic analysis, Bayesian inference, Multi-chain Markov chain Monte Carlo, Conscious detection, Compositional heterogeneity

\section{Background}

Phylogenetic analysis keeps an important role to understand current research in the biological processes and detect theory in evolution of natural selection. We extract the biological data via modeling features, and then compare these characteristics to study the biological evolution between species. The evolutionary relationship between species is generally reflected in the form of phylogenetic trees. Phylogenetic analysis can help to understand the evolutionary history of biological process, and become important data source for the development of large scale genomic data [1].

*Correspondence: fguo@tju.edu.cn

'School of Computer Science and Technology, Tianjin University, 92 Weijin

Road, Nankai District, Tianjin, People's Republic of China

Full list of author information is available at the end of the article
Many methods for constructing the phylogenetic tree, are based on optimization criteria, such as maximum parsimony, maximum likelihood and minimum evolution. Maximum parsimony (MP) approach $[2,3]$ examines all possible topologies or a certain number of topologies, which are likely to choose real phylogenetic tree or approximate phylogenetic tree with fewest evolutionary changes. Maximum likelihood (ML) approach $[4,5]$ tries to estimate trees by formulating a probabilistic model of evolution and applying known statistical method. It involves that phylogenetic tree yields the highest probability of evolutionary relationship. Minimum evolution (ME) approach [6] searches for the phylogenetic tree that minimizes total branch lengths. It is based on the assumption 
that the phylogenetic tree with smallest branch lengths is most likely to be the true one.

The correlation model of phylogenetic analysis assumes that phylogenetic trees are built on homogeneous data [7-10]. However, there exists a large deviation in the presence of heterogeneous data. As early as twenty years ago, there is first computational method [11] to detect heterogeneity problem, which makes people to doubt the credibility of phylogenetic analysis. Later, Markov model [12] of DNA sequence is used in the system development. Jukes-Cantor model [13] has been improved and taken into account unequal nucleotide compositions, different rates of changes from one nucleotide to another, variations in the form of invariant sites, and discrete gamma-distributed rates of variable sites. At the same time, researchers realize that the process of evolution would be different because of various evolutionary trees. It is obvious that the global rate can be often observed in fast and slow evolutionary species.

In this paper, we use maximum likelihood and Bayesian inference method to establish phylogenetic trees; multi-chain Markov chain Monte Carlo sampling method can be used to select optimal phylogenetic tree, resolving local optimum problem. We use two different instantaneous rate matrices, which is symmetrical and implies time-reversibility. We allow more than one composition vector to model compositional heterogeneity, because the overall model is tree-heterogeneous. The analysis is not reversible, and the likelihood depends the position of root. Compared to bootstrapping, Markov chain Monte Carlo yields a much larger sample of trees in the same computational time.

The correlation model of phylogenetic analysis assumes that phylogenetic trees are built on homogeneous data, however there exists a large deviation in the presence of heterogeneous data. The sample of trees produced by Markov chain Monte Carlo is highly auto-correlated, whereas many fewer bootstrapping replicates are sufficient. We make a conscious detection of phylogenetic tree produced by multi-chain Markov chain Monte Carlo sampling, analyzing multiple sampling and comparing different samples obtained from estimated values. We use conscious detection to solve compositional heterogeneity. Our method is evaluated on two sets of experimental data, a group of bacterial $16 \mathrm{~S}$ ribosomal RNA gene data, and a group of genetic data with five homologous species. Our method can obtain accurate phylogenetic tree on the homologous data, and also detect the compositional heterogeneity of experimental data. We provide an efficient method to enhance the accuracy of generated phylogenetic tree.

\section{Method}

We construct a phylogenetic tree for a set of DNA sequences. Our method generally contains following processes: aligning sequence [14-16], building phylogenetic trees, and selecting phylogenetic tree.

\section{Aligning sequence}

The genetic information storage location has some differences on distinct species, such as information length and carrier of genetic information. These differences will affect our subsequent analysis. Therefore, we should arrange all possible similar sites in the same position, via a progressive algorithm of multiple sequence alignment. We adopt representational evolutionary multiple sequence alignment algorithm, called ClustalW [17-19]. It displays the alignment score, in form of identities, similarities and differences, and a guide tree of evolutionary relationship between aligned sequences.

\section{Building phylogenetic trees}

The phylogenetic tree consists of many nodes and branches, where the node represents a taxon, namely species or sequence; the branch represents the evolutionary relationship between species [20,21]. All nodes are divided into external nodes and internal nodes. In general, the external node represents actual observed taxon, the internal node represents location of evolutionary event.

\section{Phylogeny model}

Given the genetic information, we need the specific phylogeny model to predict evolutionary tree. First, we use the substitution model in terms of conversion rate. In general, the instantaneous conversion matrix is expressed as follows.

$$
Q=\left(\begin{array}{cccc}
-\mu\left(a \pi_{C}+b \pi_{G}+c \pi_{T}\right) & \mu a \pi_{C} & \mu b \pi_{G} & \mu c \pi_{T} \\
\mu g \pi_{A} & -\mu\left(g \pi_{A}+d \pi_{G}+e \pi_{T}\right) & \mu d \pi_{G} & \mu e \pi_{T} \\
\mu h \pi_{A} & \mu j \pi_{C} & -\mu\left(h \pi_{A}+j \pi_{C}+f \pi_{T}\right) & \mu f \pi_{T} \\
\mu i \pi_{A} & \mu k \pi_{C} & \mu l \pi_{G} & -\mu\left(i \pi_{A}+k \pi_{C}+l \pi_{G}\right)
\end{array}\right)
$$


where this matrix specifies the rate of change from nucleotide $i$-row to nucleotide $j$-column. The nucleotides are in the order $A, C, G, T$. The stationary frequencies of nucleotides $\left(\pi_{A}, \pi_{C}, \pi_{G}, \pi_{g}\right)$ are obtained by letting the substitution process run for a very long time.

The instantaneous conversion rate matrix describes the ratio of substitutions in a short period of time, but we need to calculate probabilities of changes in a certain period of time. Then, the probability matrix can be calculated as follows.

$$
P(t)=e^{Q t}
$$

where $Q$ is the instantaneous rate matrix, $t$ is the branch length.

For a variety of evolutionary trees, we can calculate the likelihood of each phylogenetic tree. We need to consider the transformation between one external node and one internal node, and also consider the transformation between two internal nodes. For a specific site, we can calculate the likelihood of phylogenetic tree as follows.

$$
L=\sum_{y} \pi_{y_{2 s-1}} \prod_{k=1}^{s} p_{y_{\sigma(k)}, x_{k}}\left(v_{k}\right) \prod_{k=s+1}^{2 s-2} p_{y_{\sigma(k)}, y_{k}}\left(v_{k}\right)
$$

where $x$ and $y$ are the external node and the internal node, respectively. $\sigma(k)$ is the prefix index of $k, v_{k}$ is the branch length between $y_{\sigma(k)}$ and $x_{k} / y_{k}$. External nodes are $s$ input sequences, that is, $s$ species; according to the graph theory, we can get a total of $2 s-1$ internal nodes.

\section{Log-likelihood}

We assume that all sites are independent with each other. We can calculate the likelihood of each site [22], and then multiply them together to get final likelihood of phylogenetic tree.

We put all possible permutations, and then calculate the likelihood of all possibilities. For a specific site, the likelihood is the sum possibility of all internal nodes, denoted by $L_{j}$. The likelihood of all sites can be calculated as follows.

$$
\ln L=\sum_{j=1}^{N} L_{j}
$$

where $N$ refers to the length of sequence and the total number of sites.

\section{Bayesian inference}

We can use Bayesian inference [23] to produce the posterior probability of $i$-th phylogenetic tree, $\tau_{i}$, as follows.

$$
f\left(\tau_{i} \mid X\right)=\frac{f\left(X \mid \tau_{i}\right) f\left(\tau_{i}\right)}{\sum_{j=1}^{B(s)} f\left(X \mid \tau_{j}\right) f\left(\tau_{j}\right)}
$$

where $f\left(\tau_{i} \mid X\right)$ is the posterior probability of $\tau_{i}, f\left(X \mid \tau_{i}\right)$ is the likelihood of $\tau_{i}$, and $f\left(\tau_{i}\right)$ is the prior probability of $\tau_{i}$. $B(s)$ is the number of all possible trees.

\section{Selecting phylogenetic tree}

Typically, the posterior probability of phylogenies cannot be calculated analytically, but it can be approximated by sampling phylogenetic trees from the posterior probability distribution.

\section{Markov chain Monte Carlo}

Markov chain Monte Carlo (MCMC) [24] can be used to sample phylogenies according to their posterior probabilities. The Metropolis-Hastings-Green (MHG) algorithm is an MCMC method that has been used successfully to approximate posterior probabilities of trees. MHG algorithm constructs a Markov chain with the stationary frequency of posterior probability. The current state is denoted as $\tau$, and a new state is proposed as $\tau^{\prime}$. The new state is accepted with probability as follows.

$$
\begin{aligned}
R & =\min \left(1, \frac{f\left(\tau^{\prime} \mid X\right)}{f(\tau \mid X)} \times \frac{f\left(\tau \mid \tau^{\prime}\right)}{f\left(\tau^{\prime} \mid \tau\right)}\right) \\
& =\min \left(1, \frac{f\left(X \mid \tau^{\prime}\right) f\left(\tau^{\prime}\right) / f(X)}{f(X \mid \tau) f(\tau) / f(X)} \times \frac{f\left(\tau \mid \tau^{\prime}\right)}{f\left(\tau^{\prime} \mid \tau\right)}\right) \\
& =\min \left(1, \frac{f\left(X \mid \tau^{\prime}\right)}{f(X \mid \tau)} \times \frac{f\left(\tau^{\prime}\right)}{f(\tau)} \times \frac{f\left(\tau \mid \tau^{\prime}\right)}{f\left(\tau^{\prime} \mid \tau\right)}\right)
\end{aligned}
$$

One important problem of MCMC method is that we can only get the local optimal result, but not the global optimum. As shown in Fig. 1, if the current state is at the peak of $T_{1}$, because of the jump decision, the probability of next state must be less than one of current state, so MCMC method may get $T_{1}$, but miss better $T_{2}$.

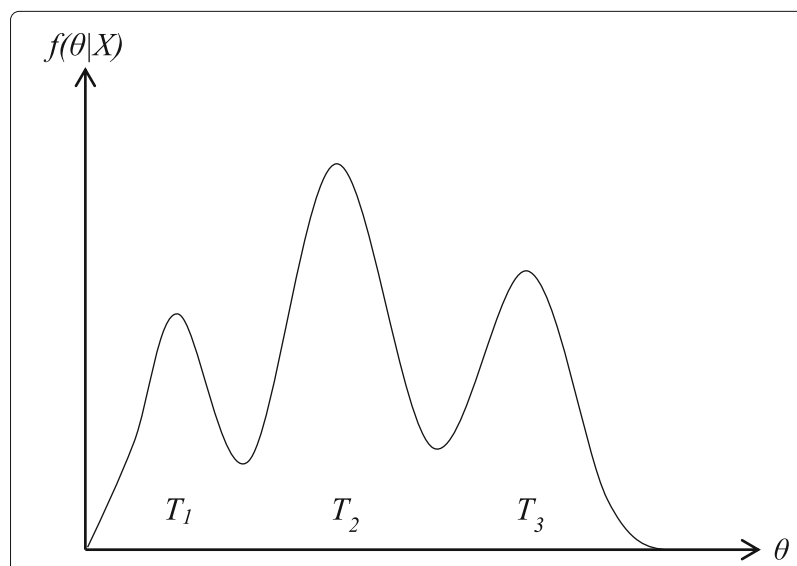

Fig. 1 Markov Chain Monte Carlo method can only get the local optimal result in range $T_{1}$ or $T_{3}$ 


\section{Multi-chain Markov chain Monte Carlo}

When the distribution becomes flat, Multi-Chain Markov Chain Monte Carlo (MCMCMC) is easy to get down from the peak of local optimum, and then try to get more states. We set a cold chain, and rest of heat chains obtained by heat values. The heat value is obtained as follows.

$$
\beta_{i}=\frac{1}{1+c(i-1)}
$$

where $c$ is the heat coefficient according to the specific experimental data, $i$ is the chain number. The state value of $i$-chain is calculated as $f_{i}(s)=f_{1}(s)^{\beta_{i}}$. Easy to see, the distribution is more gentle, as shown in Fig. 2.

Exchange occurs between two selected chains, and the exchange rate is determined as follows.

$$
R=\frac{f_{i}\left(s_{j}\right) f_{j}\left(s_{i}\right)}{f_{i}\left(s_{i}\right) f_{j}\left(s_{j}\right)}
$$

where $s$ is the state of chain, $f(s)$ is the state $s$ corresponding to the state value in the special chain. When $R$ is more than or equal to 1 , it must be exchanged; when $R$ is less than 1 , it may be exchanged with probability value.

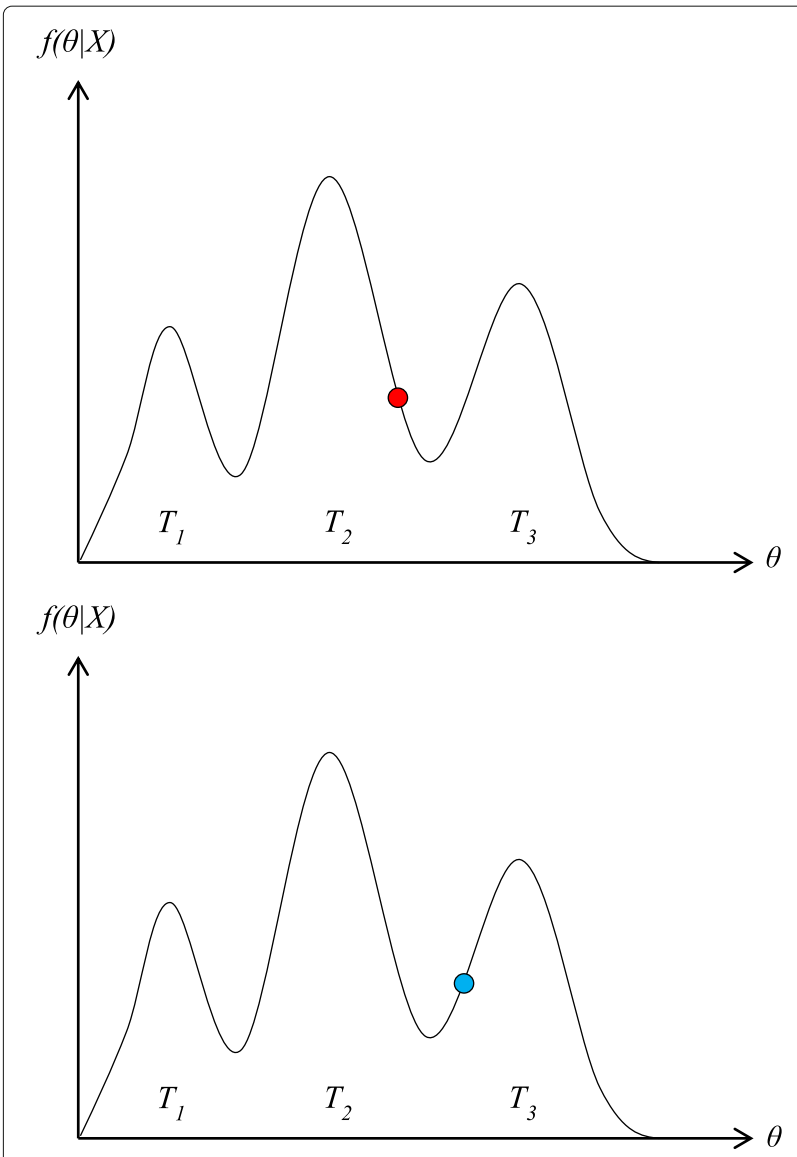

Fig. 2 Cold Chain and Heat Chain for obtaining the global optimum: red point is used for heat status and blue point is used for cold status

\section{Conscious detection}

The correlation model of phylogenetic analysis [9] assumes that phylogenetic tree is built on homogeneous data, therefore there exists a large deviation in the presence of heterogeneous data. We use conscious detection to solve compositional heterogeneity. We make a conscious detection of phylogenetic tree, analyze multiple sampling, and compare different samples obtained from estimated values. We extract the partial data from original data and form a new data set. Hundreds of data sets are used to generate different phylogenetic trees, and then get the support rate of different branches in the phylogenetic tree generated by actual data.

For $m \times n$ data set matrix, we select a random number from 1 to $n$, and obtain the column corresponding to this random number as re-sampling data for the first column; then repeat the above step to obtain re-sampling data of the second column, and so on. After $N$-loops selection, we get the final data set with same length of the original data set. For obtained data set, we analyze the phylogenetic tree according to phylogenetic analysis. Finally, we get $N$ phylogenetic trees and their posterior probabilities, and analyze the genetic information.

\section{Results and discussion}

Our method is evaluated on two sets of experimental data, a group of bacterial $16 \mathrm{~S}$ ribosomal RNA gene data, and a group of genetic data with five homologous species.

\section{Experimental environment}

We use Think Station S30 Workstation, and all programs are carried out on Ubuntu 14.04 64bit operating system, Intel Xeon E5-2620, 6 core 12 threads A-2 processor, 32G DDR3 1333MHz memory. We also use experiment softwares, such as multiple sequence alignment on CLUSTALX $2.0[25,26]$ and simulation test on JMODELTEST 2.17. The experimental data source is from National Center for Biotechnology Information (NCBI) database.

\section{Compositional heterogeneity in bacterial $16 \mathrm{~S}$ genes}

Our development system is applied to a problematic data set of bacterial 16S genes [27]: Deinococcus, Thermus,

Table 1 Bacterial 16S genes: Deinococcus, Thermus, Bacillus, Thermotoga, and Aquifex

\begin{tabular}{lll}
\hline Organism & Accession & Type \\
\hline Thermus thermophilus & NR_037066 & complete sequence \\
Bacillus subtilis & NR_102783 & complete sequence \\
Thermotoga maritima & NR_029163 & complete sequence \\
Aquifex pyrophilus & NR_029172 & partial sequence \\
Deinococcus radiodurans & NR_074411 & complete sequence \\
\hline
\end{tabular}




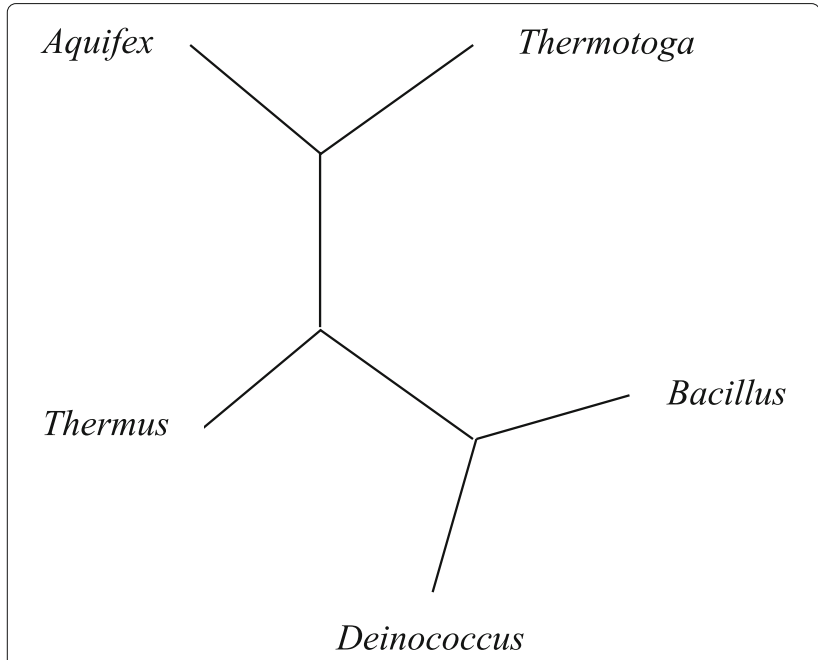

Fig. 3 Predicted evolutionary tree on Bacterial 16S Genes

Bacillus, Thermotoga, and Aquifex. Specific information is shown in Table 1.

Our method produces the phylogenetic tree on $16 \mathrm{~S}$ genes. We get prediction result with a tree (Deinococcus, (Aquifex, Thermotoga), (Thermus), Bacillus), as shown in Fig. 3. As we can see, Thermotoga and Aquifex are connected together, Bacillus and Deinococcus are connected together.

However, other biological evidence, according to their actual evolutionary relationship, should introduce actual phylogenetic tree ((Aquifex, Thermotoga), (Deinococcus, Thermus), Bacillus), as shown in Fig. 4.

\section{Conscious detection}

Here, we re-sample 100 groups of data set, and construct one phylogenetic tree for each group of data set.

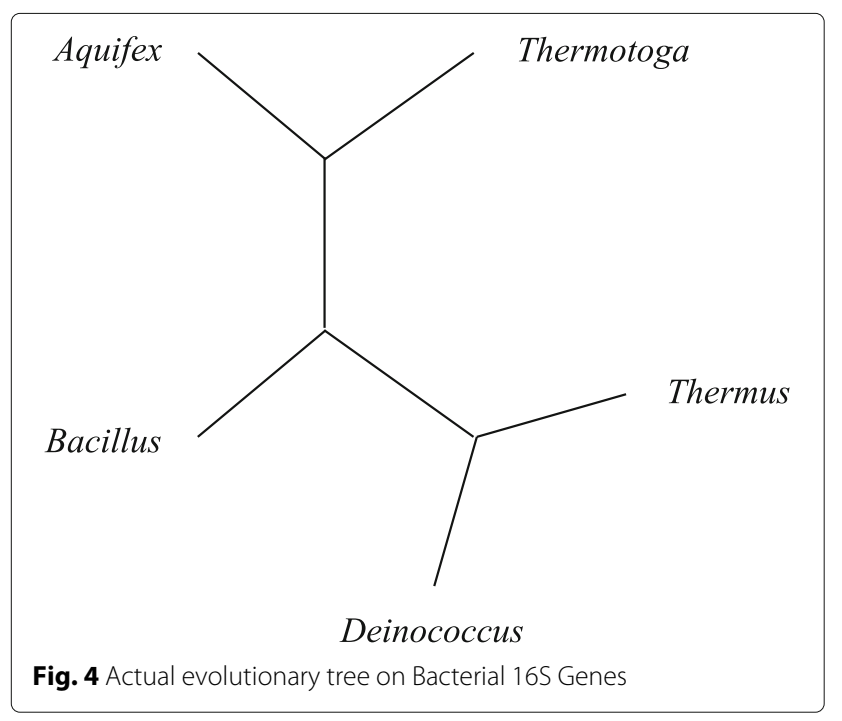

Table 2 Experiment results of our method with conscious detection on bacterial 165 genes

\begin{tabular}{ll}
\hline Posterior probability & Experimental groups \\
\hline $100 \%$ & 17 \\
{$[95 \%, 100 \%)$} & 28 \\
{$[80 \%, 95 \%)$} & 12 \\
{$[50 \%, 80 \%)$} & 10 \\
\hline
\end{tabular}

Experiment results on 67 groups of data set are the same with their actual evolutionary relationship, as shown in Table 2. Based on conscious detection, we can correct the experimental data, in order to get the actual phylogenetic tree.

\section{Homologous experiment}

We adopt homologous gene sequences to construct the evolutionary tree, and find out evolutionary relationship. We use five species of albumin and c-myc mRNA genes [28]: fish(Actinoptergyii, Salmo salar), frogs(Amphibia, Xenopus laevis), birds(Aves, Gallus gallus), rodents(Rodentia, Rattus norvegicus) and humans(Primates, Homo sapiens), as listed in Table 3.

Our method produces similar experiment results on albumin and c-myc mRNA genes. We get result with a tree (frog, (human, rodent), (bird), fish), as shown in Fig. 5. As we can see, human and rodent are connected together, frog and fish are connected together. Experiment results on albumin and c-myc mRNA genes are the same with their actual evolutionary relationship.

\section{Xanthine dehydrogenase from drosophila}

We analyze the root of Drosophila saltans and Drosophila willistoni groups, as outgroup rooting with the $\mathrm{Xdh}$ gene [29]. Based on morphology, we got the most credible root as shown in the root position $r_{1}$ in Fig. 6, as well as based on deletion of an intron in the willistoni group-specific Adh gene. The outgroup is D. virilis, D. pseudoobscura and D. melanogaster. When only the ingroup is used, an acceptable phylogeny can be generated, which is consistent with the known relationships derived from morphological characters. When outgroup taxa are used in the

Table 3 Homologous data of albumin genes and c-myc mRNA genes

\begin{tabular}{llll}
\hline Species & Organism & Accession (albumin) & $\begin{array}{l}\text { Accession } \\
\text { (c-myc mRNA) }\end{array}$ \\
\hline Actinoptergyii & Salmo salar & X52397 & M13048 \\
Amphibia & Xenopus laevis & M18350 & M14455 \\
Aves & Gallus gallus & X60688 & M20006 \\
Rodentia & Rattus norvegicus & $\mathrm{J} 00698$ & Y00396 \\
Primates & Homo sapiens & L00132 & V00568 \\
\hline
\end{tabular}




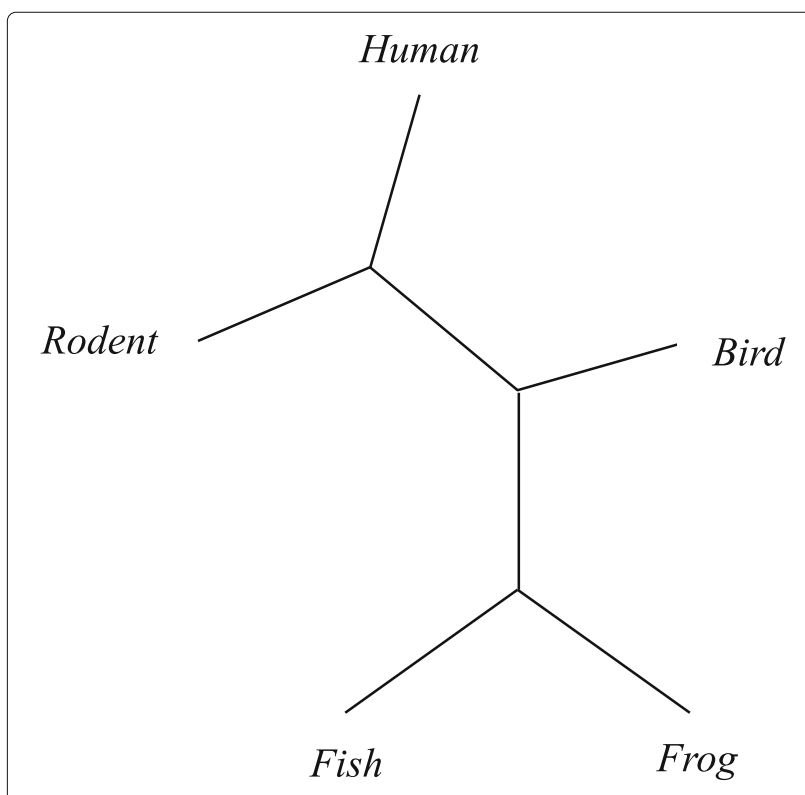

Fig. 5 Evolutionary tree on albumin and c-myc mRNA genes

analysis, depending on different model or method, the ingroup's root position became unstable. This situation is resulted by the compositional differences, especially the ones between ingroup and outgroup taxa.

Four different roots indicated by positions $r_{1}-r_{4}$ in Fig. 6 , the points where the outgroup attach to the ingroup on, are found by various methods. Here, the entire analysis's overall root and the outgroup root position can be

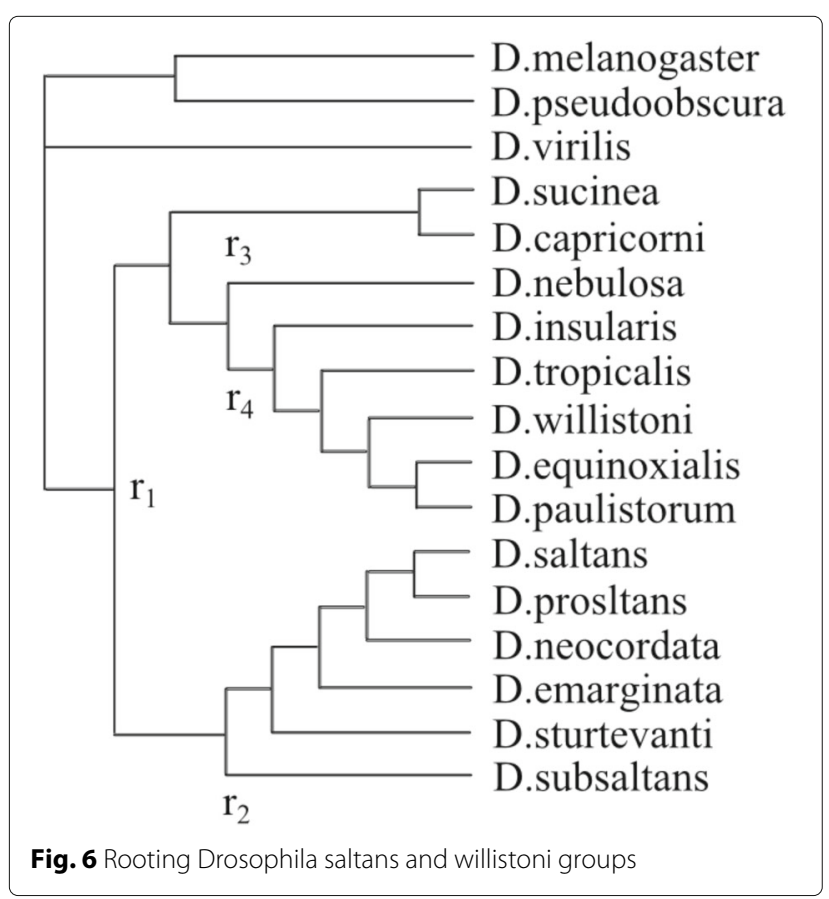

distinguished from each other, numbered as in Fig. 6. When accommodating the heterogeneous composition, this model can recover the outgroup root position $r_{1}$. A distance-based analysis can overcome compositional heterogeneity, finding the preferred root position $r_{1}$. We produce on these data to choose a model using the tree rooted at position $r_{1}$, with the expectation that our choice of model is independent on other roots. A search for the GTR+SS model using PAUP finds a tree rooted at position $r_{2}$. A Bayesian analysis using MrBayes also finds a tree rooted at position $r_{2}$.

\section{Conclusions}

In our paper, maximum likelihood, Bayesian inference method and multi-chain Markov chain Monte Carlo sampling are used to build and select global optimal phylogenetic tree. And also, compositional heterogeneity problem is solved by using conscious detection. When evaluated on two sets of experimental data, our method is efficient and accurate to generate phylogenetic tree and detect the compositional heterogeneity.

\section{Abbreviations}

DNA: Deoxyribonucleic acid; RNA: Ribonucleic acid; mRNA: Messenger RNA; MP: Maximum parsimony; ML: Maximum likelihood; ME: Minimum evolution; MCMCMC: Multi-Chain Markov Chain Monte Carlo; MCMC: Markov chain Monte Carlo; MHG: Metropolis-Hastings-Green

\section{Acknowledgements}

Not applicable.

\section{Funding}

This research and this article's publication costs are supported by a grant from the National Science Foundation of China (NSFC 61402326), Peiyang Scholar Program of Tianjin University (no. 2016XRG-0009), and the Tianjin Research

Program of Application Foundation and Advanced Technology (16JCQNJC00200).

\section{Availability of data and material}

Not applicable.

\section{About this supplement}

This article has been published as part of BMC Systems Biology Volume 11 Supplement 4, 2017: Selected papers from the 10th International Conference on Systems Biology (ISB 2016). The full contents of the supplement are available online at https://bmcsystbiol.biomedcentral.com/articles/ supplements/volume-11-supplement-4.

\section{Authors' contributions}

$Z Z$, KG and FG conceived the study. KG and FG performed the experiments and analyzed the data. ZZ, GP and FG drafted the manuscript. All authors read and approved the manuscript.

Ethics approval and consent to participate Not applicable.

\section{Consent for publication}

Not applicable.

\section{Competing interests}

The authors declare that they have no competing interests. 


\section{Author details}

${ }^{1}$ School of Computer Science and Technology, Tianjin University, 92 Weijin Road, Nankai District, Tianjin, People's Republic of China. ${ }^{2}$ Department of Computer Science and Engineering, University of South Carolina, Columbia, USA.

\section{Published: 21 September 2017}

\section{References}

1. Baxevanis AD, Ouellette BF. Bioinformatics: a practical guide to the analysis of genes and proteins: John Wiley \& Sons; 2004.

2. Eck RV, Dayhoff MO. Atlas of protein sequence and structure. Washington: National Biomedical Research Foundation; 1966.

3. Fitch WM. Toward defining the course of evolution: Minimum change for a specific tree topology. Syst Biol. 1971;20(4):406-16.

4. Felsenstein J. Evolutionary trees from dna sequences: A maximum likelihood approach. J Mol Evol. 1981;17(6):368-76.

5. Yang Z. Maximum likelihood phylogenetic estimation from DNA sequences with variable rates over sites: approximate methods. J Mol Evol. 1994;39(3):306-14.

6. Edwards AWF, Cavalli-Sforza LL. Reconstruction of evolution. Heredity. 1963;18:553.

7. Saitou N, Nei M. The neighbor-joining method: a new method for reconstructing phylogenetic trees. Mol Biol Evol. 1987;4(4):406-25.

8. Tamura K, Peterson D, Peterson N, Stecher G, Nei M, Kumar S. Mega5: molecular evolutionary genetics analysis using maximum likelihood, evolutionary distance, and maximum parsimony methods. Mol Biol Evol. 2011;28(10):2731-9.

9. Ronquist F, Huelsenbeck JP. Mrbayes 3: Bayesian phylogenetic inference under mixed models. Bioinformatics. 2003;19(12):1572-4.

10. Stamatakis A. Raxml-vi-hpc: maximum likelihood-based phylogenetic analyses with thousands of taxa and mixed models. Bioinformatics. 2006;22(21):2688-90.

11. Lockhart $P$, Steel $M$, Hendy $M$, Penny D. Recovering evolutionary trees under a more realistic model of sequence evolution. Mol Biol Evol. 1994;11:605-12.

12. Larget B, Simon DL. Markov chain monte carlo algorithms for the bayesian analysis of phylogenetic trees. Mol Biol Evol. 1999;16:750-9.

13. Jukes TH, Cantor CR, Munro HN. Evolution of protein molecules. Mammal Protein Metab. 1969;3(21):132.

14. Gibbs AJ, Mcintyre GA. The diagram, a method for comparing sequences. Eur J Biochem. 1970;16(1):1-11.

15. Smith TF, Waterman MS. Identification of common molecular subsequences. J Mol Biol. 1981;147(1):195-7.

16. Needleman SB, Wunsch CD. A general method applicable to the search for similarities in the amino acid sequence of two proteins. J Mol Biol. 1970;48(1):443-53.

17. Higgins DG, Sharp PM. Clustal: a package for performing multiple sequence alignment on a microcomputer. Gene. 1988;73(1):237-44.

18. Higgins DG, Bleasby AJ, Fuchs R. Clustal v: improved software for multiple sequence alignment. Comput Appl Biosci CABIOS. 1992;8(2):189-91.

19. Larkin MA, Blackshields G, Brown NP, Chenna R, McGettigan PA, McWilliam H, Valentin F, Wallace IM, Wilm A, Lopez R, Thompson JD, Gibson TJ, Higgins DG. Clustal w and clustal $x$ version 2.0. Bioinformatics. 2007;23(21):2947-8.

20. Ranwez V, Gascuel O. Improvement of distance-based phylogenetic methods by a local maximum likelihood approach using triplets. Mol Biol Evol. 2002;19(11):1952-63.

21. Fitch WM, Margoliash E. Construction of phylogenetic trees. Science. 1967;155(3760):279-84.

22. Lewis PO. A likelihood approach to estimating phylogeny from discrete morphological character data. Syst Biol. 2001;50(6):913-25.

23. Swofford DL. PAUP (version 3.0): phylogenetic analysis using parsimony. III Nat Hist Surv Champaign, III. 1989;9.

24. Larget B, Simon D. Markov chasin monte carlo algorithms for the bayesian analysis of phylogenetic trees. Mol Biol Evol. 1999;16(6):750.

25. Jeanmougin F, Thompson JD, Gouy M, Higgins DG, Gibson TJ. Multiple sequence alignment with clustal x. Trends Biochem Sci. 1998;23(10): 403-5.

26. Thompson JD, Gibson TJ, Plewniak F, Jeanmougin F, Higgins DG. The clustal_x windows interface: Flexible strategies for multiple sequence alignment aided by quality analysis tools. Nucleic Acids Res. 1997;25(24): 4876-82.

27. Foster PG. Modeling compositional heterogeneity. Syst Biol. 2004;53(3): 485-95.

28. Huelsenbeck JP, Ronquist F. Mrbayes: Bayesian inference of phylogenetic trees. Bioinformatics. 2001;17(8):754-5. https://academic.oup.com/ bioinformatics/article/17/8/754/235132/MRBAYES-Bayesian-inferenceof-phylogenetic-trees.

29. Tarrio R, Rodriguez-Trelles F, Ayala FJ. Tree rooting with outgroups when they differ in their nucleotide composition from the ingroup: The drosophila saltans and willistoni groups, a case study. Mol Phylogenet Evol. 2000;16(3):344-9. doi:10.1006/mpev.2000.0813.

\section{Submit your next manuscript to BioMed Central and we will help you at every step:}

- We accept pre-submission inquiries

- Our selector tool helps you to find the most relevant journal

- We provide round the clock customer support

- Convenient online submission

- Thorough peer review

- Inclusion in PubMed and all major indexing services

- Maximum visibility for your research

Submit your manuscript at www.biomedcentral.com/submit
Biomed Central 